\title{
Frequency dependence of phonon-polariton damping in lithium niobate
}

\author{
U. T. Schwarz and Max Maier \\ Naturwissenschaftliche Fakultät II - Physik, Universität Regensburg, D-93040 Regensburg, Germany
}

(Received 27 July 1995)

\begin{abstract}
We have measured the gain for stimulated Raman scattering from phonon polaritons of the $256 \mathrm{~cm}^{-1} A_{1}$ mode of $\mathrm{MgO}: \mathrm{LiNbO}_{3}$ as a function of polariton frequency. The comparison with calculations provides new information on the damping of phonon polaritons, in particular on the propagation of the polaritons out of the excitation volume, their coupling to low-frequency excitations, and the frequency dependence of the damping constant of the optical phonon mode, which is in agreement with the anharmonic decay of the optical phonon into two acoustic phonons and the scattering at crystal defects.
\end{abstract}

The study of the properties of phonon polaritons provides fundamental insight in the nature of light-matter interaction. Investigations of the damping of polaritons are a powerful tool to gain information on the material excitations to which light is coupled. Recently, it has been shown that lowfrequency phonon polaritons can be excited with femtosecond light pulses by the optical Cerenkov effect ${ }^{1}$ and by impulsive stimulated Raman scattering. ${ }^{2-4}$ In $\mathrm{LiNbO}_{3}$ (Ref. 3) and $\mathrm{LiTaO}_{3}$ (Ref. 4) information on the polariton damping and the coupling of other phonon modes to the polariton mode has been obtained. Particularly lithium niobate has enormous merits in nonlinear optics and electro-optics. Phonon polaritons with high frequencies $\left(>600 \mathrm{~cm}^{-1}\right)$ have been excited by superposing two short light pulses with appropriate frequencies and detected by time-delayed coherent anti-Stokes Raman scattering. ${ }^{5,6}$ From these investigations information on the propagation characteristics and the decay time of the high-frequency polaritons has been obtained.

In this paper, we study the damping of the polaritons with an experimental method which is suitable for polaritons with arbitrary frequencies. We measured the peak value and width of the gain for stimulated Raman scattering from the phonon polaritons of the $256 \mathrm{~cm}^{-1} A_{1}$ mode of $\mathrm{MgO}$-doped $\mathrm{LiNbO}_{3}$, which was used because of its higher threshold for optical damage. ${ }^{7}$ The dependence of the Raman gain on polariton frequency provides new information on the damping of the polaritons. The propagation of polaritons out of the excitation volume and their coupling to low-frequency excitations are important damping mechanisms for polariton frequencies smaller than 100 and $200 \mathrm{~cm}^{-1}$, respectively. Of special importance is the region of high polariton damping from 200 to $256 \mathrm{~cm}^{-1}$, where the observed frequency dependence of the polariton damping is described by a model for the anharmonic decay of its phonon part into two acoustic phonons and its scattering at crystal defects. To our knowledge, these are the first results on the frequency dependence of the anharmonic decay of the phonon part of the polaritons. Previously, either the temperature dependence of the anharmonic decay of optical phonons or the frequency dependence of the anharmonic decay of acoustic phonons has been investigated.

Polaritons with wave number $\bar{\nu}=\bar{\nu}_{L}-\bar{\nu}_{S}$ and wave vector $\mathbf{k}=\mathbf{k}_{L}-\mathbf{k}_{S}$ are excited by superimposing in the lithium niobate crystal a pump laser beam (subscript $L$ ) and a Stokes beam (subscript $S$ ) under the phase matching angle $\theta$ [see inset in Fig. 1(b)]. The pump and Stokes beams are provided by a frequency-doubled $Q$-switched single-frequency $\mathrm{Nd}$ :YAG (YAG is yttrium aluminum garnet) laser and a dye laser, respectively, which is pumped by the third harmonic of

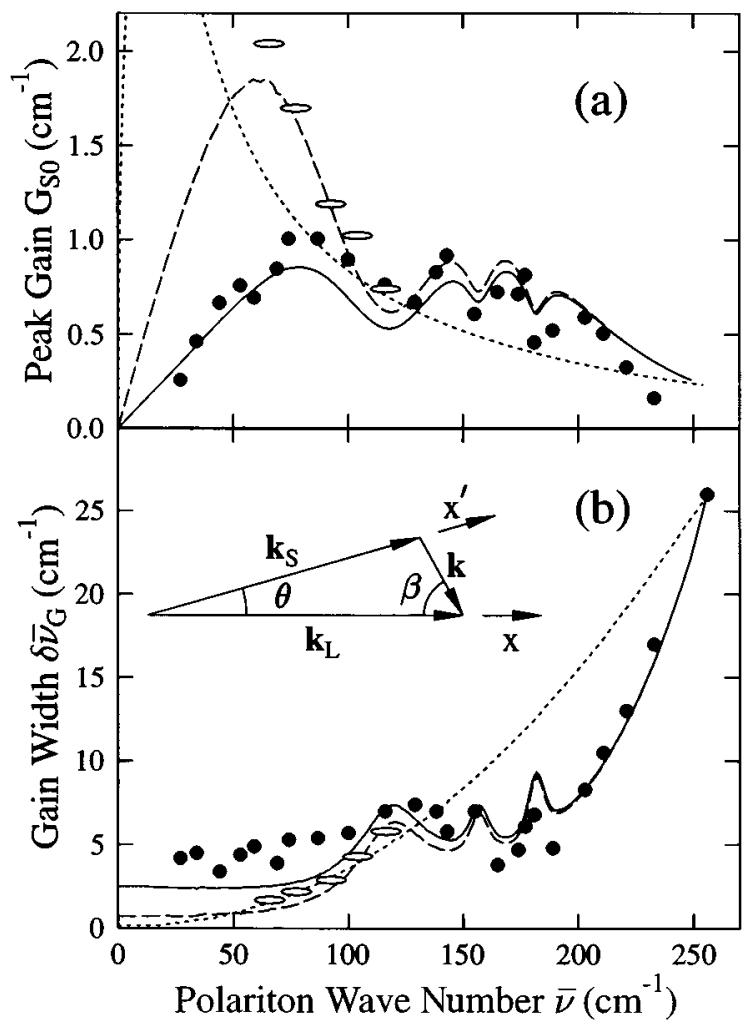

FIG. 1. Measured and calculated peak $G_{S 0}$ (a) and width $\delta \bar{\nu}_{G}$ (b) of the stimulated polariton gain curve versus polariton wave number $\bar{\nu}$. The circles and ellipses correspond to the experimental points measured with circular and elliptical light beams, respectively. The dotted lines represent the single polar phonon mode model for infinite plane waves. The solid and dashed lines represent calculations for the circular and elliptical beams, respectively, where the propagation of the polaritons out of the excitation volume, the coupling to low-frequency excitations, and the wavenumber dependence of the TO phonon damping have been taken into account. 
the Nd:YAG laser. The Stokes beam was amplified in the crystal by stimulated polariton scattering. The power of the incident and amplified Stokes pulses and the pump laser pulse, $P_{S i}(t), P_{S a}(t)$, and $P_{L}(t)$, were measured with the same photocell, a transient digitizer (risetime of the detection system about $1 \mathrm{~ns}$ ), and a computer. Typical values of the amplification $A=P_{S a} / P_{S i}$, which were observed in our experiments, lie between 2 and 20 .

We are interested in the gain $G_{S}$ for stimulated polariton scattering, which is defined using the amplified and incident Stokes intensities, $I_{S a}$ and $I_{S i}$,

$$
I_{S a}\left(y^{\prime}, z^{\prime}\right)=I_{S i}\left(y^{\prime}, z^{\prime}\right) \exp \left[\int_{-\ell / 2}^{\ell / 2} G_{S}(y, z) d x^{\prime}\right],
$$

where $\ell$ is the crystal length. The Stokes and pump laser beams propagate along the $x^{\prime}$ and $x$ directions, respectively, which form an angle $\theta$ [see inset in Fig. 1(b)]. The gain $G_{S}$ is a known function of the laser intensity ${ }^{8}$ and its $y$ and $z$ dependence is determined by the radial laser intensity distribution $I_{L}(y, z)$, with $y=x^{\prime} \sin \theta+y^{\prime} \cos \theta$ and $z=z^{\prime}$. Since we measured the Stokes power in the experiments, Eq. (1) is integrated over the Stokes beam cross section, assuming a Gaussian intensity distribution of the laser beam and the incident Stokes beam with the same 1/e radius $r_{0}$. The gain (in the center of the beam and at the time of laser pulse maximum) is then calculated numerically from the measured amplification $A=P_{S a} / P_{S i}$.

We measured the amplification $A$ in $\mathrm{MgO}: \mathrm{LiNbO}_{3}$ for a certain polariton wave number $\bar{\nu}$ at the corresponding phase matching angle $\theta$. Then we tuned the Stokes wave number $\bar{\nu}_{S}$, while keeping the phase matching angle $\theta$ constant. From the measured amplification $A$ the experimental gain $G_{S}$ was determined in the way described above. The gain $G_{S}$ as a function of Stokes wave number $\bar{\nu}_{S}$ is bell shaped for constant $\theta$. We determined the peak value $G_{S 0}$ and the width $\delta \bar{\nu}_{G}$ of this gain curve. This procedure was carried out for different polariton wave numbers $\bar{\nu}$, which lie on the polariton dispersion curve, from 30 to $256 \mathrm{~cm}^{-1}$. The accuracy of the absolute value of $G_{S 0}$ and of the width $\delta \bar{\nu}_{G}$ is determined mainly by the accuracy of the measurement of the laser power and beam radius and is limited by problems with optical damage. ${ }^{7,8}$

From the phase-matching condition the angle $\beta$ between the propagation directions of the polariton and the laser beam has been calculated [see inset in Fig. 1(b)]. Since $\beta$ is of the order of $60^{\circ}$ and the polaritons propagate with about $1 / 5$ of the vacuum light velocity (for $\bar{\nu}<100 \mathrm{~cm}^{-1}$ ), they are expected to leave rapidly the excitation volume. To check the influence of the propagation of the polaritons out of the excitation volume, the gain was determined for circular pump and Stokes beams, $1 / e$ radius $r_{0}=0.46 \mathrm{~mm}$, and for focusing of the pump and Stokes beams with a cylindrical lens, which yields an elliptical beam cross section. In this case the excitation volume is wide (about $2 \mathrm{~mm}$ ) in the propagation direction of the polaritons compared to its height $(0.1 \mathrm{~mm})$. The peak pump laser intensity in the center of the beam was about the same in both cases $\left(I_{L 0}=20 \mathrm{MW} / \mathrm{cm}^{2}\right)$.

In Fig. 1(a) the peak values $G_{S 0}$ of the polariton gain curve are plotted versus polariton wave number $\bar{\nu}$ for the circular and elliptical beams (circles and ellipses, respec- tively). It is clearly seen that for polariton wave numbers $\bar{\nu}<100 \mathrm{~cm}^{-1}$ the peak gain is lower for a smaller beam diameter in the propagation direction of the polaritons (circles). For $\bar{\nu}>100 \mathrm{~cm}^{-1}$ the same results were obtained for circular and elliptical beams within the experimental accuracy. For clarity, just the circles are shown in this wavenumber region, where $G_{S 0}$ exhibits several distinct maxima and minima as a function of polariton wave number $\bar{\nu}$.

In Fig. 1(b) the width $\delta \bar{\nu}_{G}$ of the polariton gain curve, which represents a measure of the polariton damping, is plotted versus polariton wave number $\bar{\nu} . \delta \bar{\nu}_{G}$ decreases steeply, when the polariton wave number $\bar{\nu}$ is reduced below the optical phonon wave number $\bar{\nu}_{0}=256 \mathrm{~cm}^{-1}$. In addition, three maxima are observed below $200 \mathrm{~cm}^{-1}$. For small polariton wave numbers $\left(\bar{\nu}<100 \mathrm{~cm}^{-1}\right)$ the widths $\delta \bar{\nu}_{G}$ are larger for the circular beam (circles) than for the elliptical beam (ellipses), i.e., the propagation of the polaritons out of the excitation volume acts as an additional damping mechanism.

We have calculated the gain $G_{S}$ for stimulated polariton scattering from the $256 \mathrm{~cm}^{-1} A_{1}$ mode in $\mathrm{LiNbO}_{3}$ starting with the plane-wave theory of Henry and Garrett ${ }^{9}$ and Sussman. ${ }^{10}$ In the first step we used a single polar phonon mode model with an eigenfrequency of $\bar{\nu}_{0}=256 \mathrm{~cm}^{-1}$, which leads to the dielectric constant $\varepsilon=\varepsilon_{\infty}$ $+S_{0} \bar{\nu}_{0}^{2} /\left(\bar{\nu}_{0}^{2}-\bar{\nu}^{2}-i \bar{\nu} \delta \bar{\nu}_{0}\right)$. Here, $S_{0}$ and $\delta \bar{\nu}_{0}$ are the oscillator strength and damping constant, respectively, of the phonon mode. The calculated peak value $G_{S 0}$ and the width $\delta \bar{\nu}_{G}$ of the stimulated polariton gain are plotted in Figs. 1(a) and 1(b), respectively, versus polariton wave number $\bar{\nu}$ as dotted lines. It is clearly seen that the calculated curves deviate from the measured points. Therefore, further important contributions to polariton damping have been taken into account in the next steps of the calculation.

In the experiments we used light beams with finite radius $r_{0}$, where the propagating polaritons leave the excitation volume approximately after the time $r_{0} / v_{\mathrm{gr}}$, with the group velocity $v_{\mathrm{gr}}$ of the polaritons, and do not contribute further to the amplification process. We have taken this into account in a very crude model. We calculated the infrared absorption coefficient $\alpha$ from the complex dielectric constant $\varepsilon$ and added the term $v_{\mathrm{gr}} /\left(c r_{0}\right)$ to $\alpha$. The stimulated polariton gain was then calculated using the corrected value of $\alpha$. This correction is important for polariton wave numbers below $100 \mathrm{~cm}^{-1}$, where the group velocity of the polaritons is high, and other contributions to the damping are weak (see Fig. 1).

The maxima of the gain width $\delta \bar{\nu}_{G}$ and the corresponding minima of the peak gain $G_{S 0}$ in the wave number range between 100 and $200 \mathrm{~cm}^{-1}$ (see Fig. 1) suggest an increased polariton damping at the respective wave numbers. Bakker, Hunsche, and $\mathrm{Kurz}^{3}$ have identified four resonances (at 43, 80,113 , and $137 \mathrm{~cm}^{-1}$ ) in undoped $\mathrm{LiNbO}_{3}$, which were explained by assuming that the microscopic potential of the $256 \mathrm{~cm}^{-1}$ TO phonon is extremely anharmonic and consists of three wells. Qiu, Tillert, and Maier ${ }^{11}$ observed minima in the generated far-infrared energy in undoped $\mathrm{LiNbO}_{3}$ at these four wave numbers and in addition at $185 \mathrm{~cm}^{-1}$. In our experiments in $\mathrm{MgO}$ doped $\mathrm{LiNbO}_{3}$ only three prominent wave numbers with increased polariton damping were found [maxima in Fig. 1(b) and corresponding minima in Fig. 1(a) 
at 120,158 , and $\left.183 \mathrm{~cm}^{-1}\right]$, while resonances below $100 \mathrm{~cm}^{-1}$ are missing. It is difficult to explain the difference between undoped and doped $\mathrm{LiNbO}_{3}$ because the physical nature of the increased polariton damping is not quite clear. In the potential well model of Bakker, Hunsche, and Kurz ${ }^{3}$ the influence of the resonances should disappear at low temperatures, because the corresponding energy levels are depopulated. We observed, however, the increased damping also at 20 and $77 \mathrm{~K}$, in contrast to this prediction. We tentatively ascribe the increased damping to the coupling of the polariton to low-frequency phonon modes or the excitation of electrons or holes in traps ${ }^{12}$ by the polariton. The missing resonances at 43 and $80 \mathrm{~cm}^{-1}$ in $\mathrm{MgO}$-doped $\mathrm{LiNbO}_{3}$ are then explained either by a strong broadening of the corresponding phonon modes or by different electron or hole traps in doped and undoped $\mathrm{LiNbO}_{3}$.

For simplicity, we assumed in our calculation that the polaritons are coupled to three phonon modes in the way described by Wiederrecht et al. ${ }^{4}$ It can be shown ${ }^{8}$ that the mode coupling leads to a change of the damping constant $\delta \bar{\nu}_{0}$ in the dielectric constant $\varepsilon$. It can be replaced by an effective damping constant $\delta \bar{\nu}_{\text {eff }}$ which is given by

$$
\delta \bar{\nu}_{\mathrm{eff}}=\delta \bar{\nu}_{0}+\frac{1}{i \bar{\nu}} \sum_{j=1}^{3} \frac{K_{j}}{\bar{\nu}_{j}^{2}-\bar{\nu}^{2}-i \bar{\nu} \delta \bar{\nu}_{j}}
$$

where $\bar{\nu}_{j}$ and $\delta \bar{\nu}_{j}$ are the eigenfrequency and damping constant of phonon mode $j$, and $K_{j}$ represents the coupling constant of this mode.

However, there remains one severe problem. The measured width $\delta \bar{\nu}_{G}$ [closed circles in Fig. 1(b)] of the polariton gain curve decreases much more rapidly between 256 and $200 \mathrm{~cm}^{-1}$ than predicted by the calculations based on the theory of Refs. 9 and 10 [dotted line in Fig. 1(b)]. For an explanation of this result we discuss the damping of the TO phonon at $\bar{\nu}_{0}=256 \mathrm{~cm}^{-1}$. The main mechanisms of phonon damping are the decay of the TO phonon into two acoustic phonons and the scattering of the TO phonon by a thermal phonon and at crystal defects.

We used a simple theoretical model to describe the decay of the TO phonon. ${ }^{13,14}$ The dispersion relation of the phonons in $\mathrm{LiNbO}_{3}$ (Ref. 15) shows that the $256 \mathrm{~cm}^{-1} \mathrm{TO}$ phonon can decay under conservation of energy and wave vectors into two acoustic phonons with wave numbers $\bar{\nu}_{1}$ and $\bar{\nu}_{2}$ which lie in the dispersionless part of the acoustic phonon branches. For simplicity, we neglect the anisotropy of the anharmonic lattice interactions and take into account only one decay channel. Then the damping constant $\Gamma$ of the TO phonon with wave number $\bar{\nu}$ can be written as ${ }^{14}$

$$
\Gamma(\bar{\nu})=\operatorname{const} \times\left(n_{1}+n_{2}+1\right) D_{2}(\bar{\nu}) \frac{\left|\Phi\left(\bar{\nu}, \bar{\nu}_{1}, \bar{\nu}_{2}\right)\right|^{2}}{\bar{\nu} \bar{\nu}_{1} \bar{\nu}_{2}} .
$$

The phonon occupation number in thermal equilibrium is given by $n_{i}=\left[\exp \left(h c \bar{\nu}_{i} / k_{B} T\right)-1\right]^{-1}$ with $i=1,2$. The twophonon density of states $D_{2}(\bar{\nu})$ is proportional to $\bar{\nu}^{2}$. The Fourier transform $\Phi$ of the cubic anharmonic force constants is proportional to $\bar{\nu}_{1} \bar{\nu}_{2}$. It should be emphasized that for the decay of an acoustic phonon there is an additional factor $\bar{\nu}$ in $\Phi$ [see Eq. (2) in Ref. 14], which is missing in our case, because we treated the decay of an optical phonon ${ }^{8}$ whose frequency is independent of its wave vector.

We assume that the damping of the polaritons is caused by the decay of their mechanical part and apply Eq. (3) to explain our experimental results in the wave-number range from 200 to $256 \mathrm{~cm}^{-1}$. We suggest as the dominant process the decay into two acoustic phonons with $\bar{\nu}_{1}=\bar{\nu}_{2}=\bar{\nu} / 2$, where $\bar{\nu}$ is the polariton wave number. The wave-number dependence of the phonon occupation number $n$ is neglected in the narrow range between 200 and $256 \mathrm{~cm}^{-1}$. When all these assumptions are taken into account, the polariton damping constant $\Gamma(\bar{\nu})$, caused by the decay into two acoustic phonons [Eq. (3)], is proportional to $\bar{\nu}^{3}$. This is in contrast to the decay of an LA phonon, where a $\bar{\nu}^{-5}$ dependence has been observed. ${ }^{14}$

We believe that the scattering of the polariton by a thermal acoustic phonon, or vice versa, does not make a significant contribution to the polariton damping between 200 and $256 \mathrm{~cm}^{-1}$. Since in the scattering process just one acoustic phonon is involved, the wave number dependence of both the two-phonon density of states $D_{2}(\bar{\nu})$ and the Fourier transform $\Phi$ will be different from that of the decay process in which two acoustic phonons are generated. It is, however, difficult to make a quantitative estimate because of unknown phonon eigenvectors and incomplete phonon dispersion curves of $\mathrm{LiNbO}_{3}$. ${ }^{15}$

In $\mathrm{MgO}$-doped $\mathrm{LiNbO}_{3}$ the scattering of the polariton at defects represents an important contribution to polariton damping. This can be seen from the measured temperature dependence of the spontaneous Raman linewidth of the $256 \mathrm{~cm}^{-1}$ phonon of doped, undoped congruent, and undoped stoichiometric $\mathrm{LiNbO}_{3}$, where a temperatureindependent part of the Raman linewidth has been found and ascribed to phonon scattering processes at defects. ${ }^{16}$ We have fitted the temperature dependence of the phonon occupation number $n$ to the experimental results of Ref. 16 for $\mathrm{MgO}$ doped $\mathrm{LiNbO}_{3}$. Good agreement is obtained only when a temperature-independent term, caused by phonon scattering at defects, is added to Eq. (3). If we assume Rayleigh scattering to be dominant, the scattering efficiency is proportional to $\bar{\nu}^{4}$. The total polariton damping constant can be written as

$$
\delta \bar{\nu}_{0}=\Gamma_{d}\left(\bar{\nu} / \bar{\nu}_{0}\right)^{3}+\Gamma_{s}\left(\bar{\nu} / \bar{\nu}_{0}\right)^{4}
$$

$\Gamma_{d}$ and $\Gamma_{s}$ represent the contributions of the polariton decay and scattering processes, respectively. From the fit of Eq. (4) to the measured temperature dependence of the spontaneous Raman linewidth ${ }^{16}$ we obtained values of $\Gamma_{d}=17 \mathrm{~cm}^{-1}$ and $\Gamma_{s}=9 \mathrm{~cm}^{-1}$ for $\mathrm{MgO}: \mathrm{LiNbO}_{3}$ at room temperature.

We calculated the stimulated polariton gain $G_{S}$ as a function of polariton wave number $\bar{\nu}$, taking into account the propagation of the polaritons out of the excitation volume, their coupling to low-frequency phonon modes [Eq. (2)], and the wave-number dependence of the damping constant $\delta \bar{\nu}_{0}$ [Eq. (4)]. The values of the coupling and damping constants of the low-frequency phonon modes in Eq. (2) have been fitted to get good agreement between the measured points and the calculated curves. However, no fitting parameters were used for the other damping mechanisms. In particular, in Eq. (4) only parameters determined independently in other 
experiments have been introduced. It can be seen from Figs. 1(a) and 1(b) that our model describes the main features of our experimental results. Below $100 \mathrm{~cm}^{-1}$ the differences between the circular and elliptical light beams (circles and ellipses, respectively) are described reasonably well by the calculations (solid and dashed lines), where the different beam diameters and the propagation of the polaritons have been taken into account. The experimentally observed maxima and minima between 100 and $200 \mathrm{~cm}^{-1}$ are accounted for by the coupling of the polaritons to low-frequency excitations. Finally, the rapid decrease of the gain width $\delta \bar{\nu}_{G}$ [Fig. 1(b)] below $\bar{\nu}_{0}=256 \mathrm{~cm}^{-1}$ is reproduced very well by our calculations which include the anharmonic decay and the scattering at defects of the polaritons.
In conclusion, we have shown that the gain measurements of stimulated polariton scattering are a sensitive method for determining the frequency dependence of the polariton damping over a large frequency range. New information on the damping of the phonon polaritons of the $256 \mathrm{~cm}^{-1} \mathrm{TO}$ mode of $\mathrm{LiNbO}_{3}$ has been obtained. In particular, the frequency dependence of the decay of the phonon part of the polariton into two acoustic phonons has been measured for the first time and shown to be in agreement with a theoretical model.

The authors gratefully acknowledge valuable help by D. Strauch in calculating the frequency dependence of the TO phonon damping constant.
${ }^{1}$ K.P. Cheung and D.H. Auston, Phys. Rev. Lett. 55, 2152 (1985); D.H. Auston and M.C. Nuss, IEEE J. Quant. Electron. 24, 184 (1988)

${ }^{2}$ H.J. Bakker, S. Hunsche, and H. Kurz, Phys. Rev. Lett. 69, 2823 (1992).

${ }^{3}$ H.J. Bakker, S. Hunsche, and H. Kurz, Phys. Rev. B 50, 914 (1994).

${ }^{4}$ G.P. Wiederrecht, T.P. Dougherty, L. Dhar, K.A. Nelson, D.E. Leaird, and A.M. Weiner, Phys. Rev. B 51, 916 (1995).

${ }^{5}$ F. Vallée and C. Flytzanis, Phys. Rev. B 46, 13799 (1992).

${ }^{6}$ F. Vallée and C. Flytzanis, Phys. Rev. Lett. 74, 3281 (1995).

${ }^{7}$ J. Deng, W. Zhang, J. Wen, G. Zhang, and H. Wang, Opt. Lett. 19, 933 (1994).

${ }^{8}$ U.T. Schwarz and M. Maier (unpublished).
${ }^{9}$ C.H. Henry and C.G.B. Garrett, Phys. Rev. 171, 1058 (1968).

${ }^{10}$ S.S. Sussman, M. L. Report No. 1851, Microwave Laboratory, W. W. Hansen Laboratories of Physics, Stanford University, Stanford, California (1970).

${ }^{11}$ T. Qiu, T. Tillert, and M. Maier, Opt. Commun. 119, 149 (1995).

${ }^{12}$ K.L. Sweeney, L.E. Halliburton, D. A. Bryan, R.R. Rice, R. Gerson, and H.E. Tomaschke, J. Appl. Phys. 57, 1036 (1985).

${ }^{13}$ A.S. Pine and P.E. Tannenwald, Phys. Rev. 178, 1424 (1969).

${ }^{14}$ K. Okubo and S.-I. Tamura, Phys. Rev. B 28, 4847 (1983).

${ }^{15}$ M.R. Chowdhury, G.E. Peckham, and D.H. Saunderson, J. Phys. C 11, 1671 (1978).

${ }^{16}$ S. Kojima, Jpn. J. Appl. Phys. 32, 4373 (1993). 